\title{
Tanulmány
}

\section{Beatrix Babett Bódi \\ La problématique de la scène et du segment dans « Farce normande » de Guy de Maupassant}

\begin{abstract}
This paper aims to present the organization of the narrative text through a structural analysis of Guy de Maupassant's Farce normande, with respect to the system of characters. The study of this short story examines the possible division of the text into sub-units at two different levels. At the first level, the textual analysis considers the elements of the surface, in connection with the division of the text into what we call scenes (scènes). At the second level, the narratological approach reflects the deep structure of the text, and offers a division into segments, using A. J. Greimas's actantial model. This study shows a possible approach for the structural analysis of a literary text, and may serve as a basis for a typology on a larger corpus.

Keywords: narratology, actantial model, short story
\end{abstract}

\section{Introduction}

Dans cet article, nous nous proposons d'examiner les relations des personnages et les rôles qu'ils remplissent dans l'intrigue d'une nouvelle de Guy de Maupassant, " Farce normande ", racontant un épisode de la vie rurale: une fête de noce qui finit par une plaisanterie traditionnelle. ${ }^{1}$ Pour mener à bien ce travail - s'agissant d'un texte narratif littéraire - , nous allons faire une étude à la fois textuelle et narratologique. En effet, cette deuxième approche nous semble pertinente puisque le personnage, étant l'un des constituants de l'histoire relatée dans une œuvre narrative, est placé au centre de la théorie et des analyses narratologiques. En revanche, la première approche, textuelle, nous paraît également nécessaire pour montrer comment les relations des personnages évoluent au cours de l'intrigue. Nous supposons que cette évolution connaît des étapes que l'on peut découvrir non seulement au niveau, plus profond, des événements, mais aussi au niveau du récit, du texte narratif même. L'examen de cette évolution nécessite donc le découpage du texte en ses unités plus petites, découpage qui permettra de suivre les changements dans les rapports des personnages.

1 La nouvelle fait partie de la section « Drames et propos rustiques » du recueil Contes et nouvelles de Guy de Maupassant (v. notre Bibliographie). 
Beatrix Babett Bódi:

La problématique de la scène et du segment dans « Farce normande » de Guy de Maupassant

Argumentum 17 (2021), 807-819

Debreceni Egyetemi Kiadó

DOI: $10.34103 /$ ARGUMENTUM/2021/43

\section{Problèmes de découpage - Précisions terminologiques}

\subsection{La notion de séquence}

En général, un texte - ainsi le texte narratif littéraire - est une unité hétérogène, ${ }^{2}$ constituée d'une série structurée de sous-unités, souvent appelées séquences. Cependant, ce terme est employé dans des acceptions diverses, quoique toujours en rapport avec la structuration du texte. Selon Martin Riegel, Jean-Christophe Pellat et René Rioul, «Un texte possède une structure globale; il est formé de parties ou de séquences dont le sens se définit par rapport à son sens global » (Riegel, Pellat \& Rioul 2009 : 1019 - c'est nous qui soulignons).

Comparée à cette définition à la fois générale et concise du concept de séquence, celle de Jean-Michel Adam est plus spécifique et en même temps plus ample. Dans une perspective pragmatique et textuelle, Adam considère le texte comme « une configuration réglée par divers modules ou sous-systèmes en constante interaction » (Adam 1992 : 21). Il établit donc plusieurs plans d'organisation complémentaires, dont l'organisation séquentielle, et il définit la notion de séquence - unité textuelle - comme « un réseau relationnel hiérarchique : grandeur décomposable en parties reliées entre elles et reliées au tout qu'elles constituent ; unité relativement autonome, dotée d'une organisation interne qui lui est propre et donc en relation de dépendance / indépendance avec l'ensemble plus vaste dont elle fait partie » (Adam 1992 : 28). Partant du caractère hétérogène du texte, Adam distingue les cinq séquences prototypiques suivantes: narrative, descriptive, argumentative, explicative et dialogale (Adam 1992: 30) qui, dans un texte, peuvent apparaître en combinaisons variées, avec, en général, la dominance de l'un de ces types de séquence. Dès la première lecture d'un texte, on peut normalement identifier les séquences, grâce à leurs propriétés spécifiques souvent manifestées jusque dans la formulation linguistique.

Sans avoir épuisé toutes les interprétations de la notion de séquence, mentionnons ici une dernière définition, celle d'Algirdas Julien Greimas, à qui nous allons encore nous référer dans cette étude. Pour lui, la séquence est une " unité linguistique du discours », plus précisément: «La séquence est [...] - et c'est là une de ses définitions possibles - une unité du discours narratif autonome, susceptible de fonctionner comme un récit, mais pouvant également se trouver intégrée, comme une de ses parties constitutives, dans un récit plus large » (Greimas 1970 : 253). On voit bien qu'ici, Greimas, en tant que narratologue, parle exclusivement du rapport étroit entre la séquence (de grandeurs variées) et le récit. ${ }^{3}$

\subsection{Scène et segment}

Étant donné la polysémie du terme séquence, nous préférons l'abandonner, malgré son emploi courant dans les analyses textuelles. D'autant plus que - comme nous l'avons suggéré plus haut - pour décrire les étapes de l'évolution des rapports qui se tissent entre les personnages, nous aurons besoin d'un double découpage - notamment un découpage à deux niveaux -, ce qui nécessitera une distinction entre deux types d'unité, chacun étant désigné par un terme qui lui est propre. En effet, nous pensons que les événements et les personnages constituent une sorte

2 «On constate que les textes réels sont généralement hétérogènes. Un texte donné ne réalise pas un seul type textuel, mais se caractérise par la combinaison de plusieurs types imbriqués » (Riegel, Pellat \& Rioul 2009: 1063). V. aussi Maingueneau (2003 : 178-180).

3 Greimas utilise ce terme aussi dans son analyse de la nouvelle Deux amis de Guy de Maupassant. V. Greimas (1976) dans notre Bibliographie. 
de structure profonde - que nous identifions ici avec l'histoire -, mais que cette structure profonde ne peut être atteinte qu'à travers la structure de surface du texte narratif ou récit. ${ }^{4}$ Nous désignerons les unités obtenues lors de l'analyse de la structure profonde par le terme segment, tandis que l'autre type d'unité, caractérisant la structure de surface, sera appelé scène. Notre choix de termes est, certes, arbitraire, mais l'important est que les deux puissent s'opposer nettement tout au long de notre travail. En tout cas, les deux notions font référence à des parties composantes qui se combinent pour former un tout, et comportent l'idée à la fois de la division, de l'unité et de l'organisation.

Notre démarche sera la suivante : après un bref examen des notions de scène et de segment, ainsi que des possibilités de leurs délimitations respectives, nous allons procéder à l'analyse, dans "Farce normande », des scènes relevées au niveau (de surface) du récit, pour passer ensuite à l'étude des segments au niveau (profond) de l'histoire, afin d'y suivre les changements dans les rapports des personnages. Pour terminer, nous montrerons également les correspondances entre les unités des deux niveaux.

\subsection{La scène - Définition et délimitation}

Tout en étant arbitraire, le choix de ce terme a été en partie motivé par son emploi dans le contexte du théâtre, car au théâtre comme dans le récit, les scènes sont déterminées par " l'unité, au moins relative, de lieu et de temps, et surtout le développement des données toutes présentes dès le début $\gg$ (Ubersfeld 1982: 210). ${ }^{5}$ Unités spatio-temporelles, et supposant la présence permanente d'un (ou d'un groupe de) personnage(s), les scènes, dans leur succession, organisent le fil des événements, réalisant, pour ainsi dire, la composition diachronique du récit. Ubersfeld souligne l'importance de la temporalité de cette composition : " le nombre et la succession des événements de la fable, le rythme de la succession des unités du texte, la longueur des unités (surtout des séquences moyennes, des scènes), donnent de la durée temporelle un sentiment différent » (Ubersfeld $1982: 203$ ).

Cependant, la notion de scène reçoit une autre interprétation - quoique toujours liée à la temporalité - dans la narratologie de Gérard Genette, qui considère la scène comme l'une des « quatre formes fondamentales du mouvement narratif » (Genette 1972 : 129), formes établies selon les rapports variables entre le temps de l'histoire et le temps du récit. ${ }^{6}$ Or, selon Genette, la scène, presque toujours " dialoguée », " réalise conventionnellement l'égalité de temps entre récit et histoire» (Genette 1972: 129), et représente traditionnellement un contenu « dramatique », « les temps forts de l'action coïncidant avec les moments les plus intenses du récit » (Genette $1972: 142$ ). ${ }^{7}$ Pour nous, cette interprétation de la scène reste importante, mais son caractère « dramatique » ne sera pas décisif. C'est qu'il peut y avoir dans un récit, comme au théâtre, des scènes qui servent plutôt à conduire seulement vers des moments intenses, de sorte que nous ne retenons comme critère pertinent que la permanence, à l'intérieur d'une même scène, de la triade espace - temps - personnages.

4 Selon la terminologie de G. Genette (1972: 72).

5 Ces paroles d'Anne Ubersfeld, définissant à l'origine non la scène mais l'acte, peuvent s'appliquer sans difficulté à la notion de scène.

6 Rappelons que chez Genette, les quatre formes fondamentales du mouvement narratif, ou plus simplement, les quatre mouvements narratifs, sont : l'ellipse, la pause descriptive, la scène et le sommaire (Genette 1972 : 129).

7 C'est en cela que la scène s'oppose au sommaire, lequel représente un contenu non dramatique, « les temps faibles [étant] résumés à grands traits et comme de très loin » (Genette 1972 : 142). 
Si ces trois coordonnées sont nécessaires pour l'identification d'une scène particulière, elles contribuent également à sa délimitation par rapport aux unités voisines semblables. En effet, les scènes, en tant qu'unités du récit, sont séparées les unes des autres par des limites que nous pouvons détecter dans le texte : les points de rupture ${ }^{8}$ sont les endroits du texte qui marquent la délimitation de ces unités, et dont les réalisations textuelles se montrent variées. Un simple changement de décor ou de temps peut annoncer le passage à une nouvelle scène; dans la formulation linguistique, cela se traduit par des compléments circonstanciels de lieu et de temps, ainsi que par l'alternance des temps verbaux. L'arrivée ou le départ d'un personnage, ainsi que le changement de la perspective narrative, avec éventuellement un changement d'éléments déictiques (pronoms personnels, adverbes), peuvent également fonctionner comme marqueurs de délimitation entre les unités textuelles. Au-delà de ces indices linguistiques, une scène se caractérise par une thématique plus ou moins homogène, que l'on peut désigner par un syntagme nominal à caractère résomptif.

\subsection{Le segment - Définition et délimitation}

Tandis que le terme scène peut avoir des connotations théâtrales, mais également convenables pour le récit, le terme segment semble avoir un sens plus abstrait (relatif à la géométrie, selon les dictionnaires). C'est ce plus haut degré d'abstraction qui nous a suggéré le choix de ce terme pour désigner les unités du niveau lui-même plus abstrait, car plus profond, de l'histoire, où se situent les événements et les personnages avec leurs relations mutuelles et changeantes. Comme les indices linguistiques "purs » ne sont plus suffisants à ce niveau-là pour identifier et délimiter les unités, il a fallu trouver un cadre théorique permettant la segmentation de l'histoire en fonction des modifications dans les rapports des personnages, ces rapports étant comme des «forces d'action » responsables de la dynamique de l'intrigue. Or, le cadre théorique le plus propice est, à notre avis, le modèle actantiel d'Algirdas Julien Greimas. Nous pensons que ce modèle, construit en 1966, n'a pas vieilli, et que sans être exclusif, il offre une bonne base pour l'analyse narratologique de l'histoire, dans les œuvres narratives, voire dramatiques. ${ }^{9}$

Rappelons brièvement que, pour décrire les rôles fondamentaux que les personnages remplissent dans le « micro-univers » d'une œuvre (Greimas 1966 : 174), le modèle opère avec six actants distribués en trois couples, dont les membres respectifs sont plus étroitement liés entre eux : sujet - objet, destinateur-destinataire, adjuvant - opposant. Ces actants abstraits sont représentés par des acteurs, terme utilisé par Greimas pour être applicable à la fois aux personnages (dans le sens traditionnel) et aux idées, émotions ou intentions qui peuvent jouer dans la configuration du micro-univers. Par ailleurs, un actant peut être réalisé par plusieurs acteurs, et inversement, un acteur peut remplir plusieurs rôles actantiels (Greimas 1966 : 177178). ${ }^{10}$ En fait, ce modèle représente les rôles constituant le micro-univers comme un spectacle achronique, avec une distribution permanente des rôles, ce qui s'explique par les types de textes ayant servi de corpus pour les premières analyses, à savoir les contes populaires et les récits

8 La notion de rupture apparaît chez Bernard Combettes. Il définit les ruptures, ou ruptures thématiques en relation avec le phénomène de la progression thématique : «les cas de ,rupture thématique” [...] vont se produire lorsque le thème d'une phrase ne peut être attaché au contexte précédent, lorsqu'on ne peut déceler un enchaînement linéaire ou un thème constant »(Combettes 1983 : 103). Cette définition, employée ici à la succession des phrases, peut être adaptée à la succession des scènes.

9 Pour ces dernières, v. les nombreuses analyses dans Ubersfeld : Lire le théâtre (1982).

10 Greimas parle à ce propos de la « manifestation syncrétique fréquente des actants » (Greimas $1966: 177)$. 
mythiques, où les personnages remplissent des rôles prédéterminés par les exigences du genre. Cependant, dans la littérature moderne, on constate une plus grande variété quant à la distribution des rôles, et à la possibilité, pour les personnages, de changer de rôle au cours de l'intrigue.

Ce sont précisément ces changements qui permettent d'identifier et de délimiter les segments de l'histoire: à chaque segment appartient un groupe de personnages, avec une certaine distribution des rôles, et dont la permanence d'un bout à l'autre assure l'unité du segment. Mais dès qu'il y a une redistribution des rôles parmi les personnages, un nouveau segment va commencer. Afin de distinguer les deux types de limites selon le niveau auquel ils se rapportent, nous allons employer ici non l'expression point de rupture, ou simplement rupture, réservée à la structure de surface du récit, mais le terme tournant, qui suggère plus fortement les transformations décisives de l'histoire. La différence entre ces deux types de coupure réside dans leur accessibilité pour le lecteur : les ruptures, phénomènes textuels, sont directement observables dès la première lecture, tandis que les tournants exigent un processus cognitif d'interprétation des rapports des personnages entre eux et à l'ensemble des événements.

\section{Analyse textuelle et narratologique de "Farce normande "}

\section{Résumé de l'histoire :}

Jean Patu, le plus riche fermier du pays épouse Rosalie Roussel, belle fille, fort courtisée par tous les partis des environs. C'est le jour du mariage, et tous les invités se rendent à la grande ferme pour dîner et fêter ensemble. Pendant le dîner, les invités font de lourdes plaisanteries et se moquent de la nuit de noces, car c'est la tradition normande. Quatre gars, des voisins, préparent alors une farce en menaçant le riche époux de lui voler des biens pendant la nuit de noces.

La nuit de noces arrive, les jeunes mariés se retirent dans leur chambre, mais soudain un coup de feu se fait entendre de loin et les dérange. Un second coup de feu retentit, et Jean quitte alors sa femme pour aller chercher les « braconniers », mais il devient victime de la farce de ses voisins, et passe la nuit dans les bois, piégé et ficelé, au lieu d'aimer sa jeune femme.

\subsection{Les scènes de la nouvelle}

La première lecture de la nouvelle nous permet de connaître l'histoire relatée et aussi de saisir le déroulement du récit, d'où se dégagent les événements constituant le fil de l'intrigue.

\subsubsection{La structure scénique}

La nouvelle raconte donc une histoire de la vie rurale : la fête des noces avec les événements qui s'y attachent, et une farce commise contre le jeune marié. Lors de cette première lecture linéaire, la composition diachronique se révèle et, sur ce fil, nous pouvons détecter six scènes, complétées par une clôture contenant les propos du jeune mari :

Et, plus tard, quand il racontait cette nuit d'épousailles, il ajoutait : « Oh ! pour une farce ! c'était une bonne farce. Ils m'ont pris dans un collet comme un lapin, les salauds, et ils m'ont caché la tête dans un sac. Mais si je les tâte un jour, gare à eux !» (67) 
et un bref commentaire généralisant de la part du narrateur :

Et voilà comment on s'amuse, les jours de noce, au pays normand. (67)

Ces propos ne se rattachent pas strictement à l'intrigue.

\subsubsection{Délimitation des scènes}

Pour définir la suite des scènes, notre tâche est de chercher les points de rupture qui marquent la délimitation entre deux scènes consécutives. La Scène 1 présente la procession qui tend vers la ferme maritale : les descriptions du paysage, de la grande ferme, des vêtements typiques - et plus tard, dans la Scène 4, de l'intérieur de la chambre des jeunes époux - dessinent un tableau de la vie rurale de l'époque, tout en créant l'espace représenté dans le récit et accentuant l'effet de réel. À la fin de la Scène 1, nous pouvons saisir un changement d'espace et de décor : les invités arrivent à la maison maritale.

Devant la porte, les femmes tapaient sur leurs robes pour en faire tomber la poussière, dénouaient les oriflammes qui servaient de rubans à leurs chapeaux, défaisaient leurs châles et les posaient sur leurs bras, puis entraient dans la maison pour se débarrasser définitivement de ces ornements.

La table était mise dans la grande cuisine, qui pouvait contenir cent personnes.

On s'assit à deux heures. (64)

Dans ce nouveau décor, le début de la Scène 2 - celle-ci représentant le dîner - est marqué également par un changement des temps verbaux et par un complément circonstanciel de temps : l'emploi du passé simple après la prédominance de l'imparfait dans le passage descriptif de la Scène 1, et à deux heures indiquent le point de rupture.

La transition entre la Scène 2 et la Scène 3 est de nouveau marquée par le changement des temps verbaux - on passe, ici aussi, de l'imparfait de description au passé simple - et par le verbe commencer, dont le sens renvoie au début d'une nouvelle scène, moments de détente et de gaieté dans une atmosphère frivole :

[...] toutes [les fermières] alors se levèrent à la suite. [...] Et les lourdes plaisanteries commencèrent. (65)

Le début de la Scène 4 - la nuit de noces - est signalé par l'adverbe de temps puis, par l'emploi du passé simple et du passé antérieur, par le changement d'espace et par la présence exclusive du couple :

Puis, quand on eut bu des barils d'eau-de-vie, chacun partit se coucher; et les jeunes époux entrèrent en leur chambre $[\ldots](66)$

La conjonction temporelle quand et le motif du coup de feu marquent le commencement de la Scène 5 :

[...] quand un coup de feu retentit au loin, dans la direction du bois des Râpées, lui sembla-t-il. (66),

scène qui introduit le soupçon d'une menace, provoquant des réactions contradictoires chez le mari. 
Les compléments circonstanciels de temps renvoient au début de la Scène 6 :

Elle attendit une heure, deux heures, jusqu'au jour. (67),

scène qui se termine enfin par le retour du jeune époux libéré.

Cependant, entre la fin de la Scène 5 - signalée par le brusque départ du mari à la chasse des «braconniers »- et le début de la Scène 6, la transition prend une forme complètement différente par rapport aux délimitations des autres scènes. En effet, celles-ci se faisaient sans heurt, conformes à l'écoulement du temps des événements de la journée et d'une partie de la nuit, tandis que maintenant, entre les deux dernières scènes, il y a dans le récit une ellipse de quelques heures : seul le laps de temps est indiqué - après coup -, et l'événement non représenté de la farce sera seulement deviné par le lecteur voyant l'état minable du mari retrouvé, ficelé des pieds à la tête. De plus, l'ellipse va de pair avec un important changement de la perspective narrative : contrairement au point de vue externe du narrateur dans les scènes précédentes, l'ellipse représente le point de vue d'un personnage, Rosalie, ignorant l'événement élidé. Le lecteur, quant à lui, ne peut que partager cette ignorance avant la révélation finale.

En résumé, nous pouvons constater que, dans cette nouvelle, la délimitation des scènes consécutives peut être exprimée par le simple changement d'espace, par l'emploi des adverbes, et plus généralement, de divers compléments circonstanciels de lieu et de temps, et encore par l'alternance des temps verbaux. Ces marqueurs sont présents au niveau de la structure de surface du texte, et peuvent s'appliquer individuellement ou en combinaisons variées.

Pour évaluer le rôle des personnages dans la délimitation des scènes, nous pouvons constater que, dans cette nouvelle, les personnages caractérisent les scènes individuelles par leur présence et leurs comportements, mais pour la majorité des scènes, ils n'en définissent pas les frontières. Cependant, dans le cas de la Scène 5, l'apparition des « braconniers » imaginaires - perceptible uniquement par les coups de feu - implique le passage à une nouvelle scène.

\begin{tabular}{|c|c|c|}
\hline Scènes & Thème de la scène & Personnages de la scène \\
\hline Scène 1 & La procession & $\begin{array}{l}\text { le jeune marié Jean Patu; la jeune mariée Rosalie } \\
\text { Roussel ; les parents ; les invités }\end{array}$ \\
\hline Scène 2 & Le dîner & $\begin{array}{l}\text { le jeune marié Jean Patu; la jeune mariée Rosalie } \\
\text { Roussel ; les parents ; les invités }\end{array}$ \\
\hline Scène 3 & $\begin{array}{l}\text { Les plaisanteries verbales à la table } \\
\text { de dîner }\end{array}$ & $\begin{array}{l}\text { le jeune marié Jean Patu; la jeune mariée Rosalie } \\
\text { Roussel; les parents; les invités, dont les quatre } \\
\text { gars }\end{array}$ \\
\hline Scène 4 & $\begin{array}{l}\text { La nuit de noces dans la chambre } \\
\text { des jeunes mariés }\end{array}$ & les jeunes mariés, Rosalie et Jean \\
\hline Scène 5 & L'apparition des « braconniers » & Rosalie et Jean ; les « braconniers » (les quatre gars) \\
\hline Scène 6 & Le mari retrouvé & Rosalie ; Jean ; les valets ; les charretiers ; les gars \\
\hline
\end{tabular}


Beatrix Babett Bódi:

La problématique de la scène et du segment dans « Farce normande » de Guy de Maupassant

Argumentum 17 (2021), 807-819

Debreceni Egyetemi Kiadó

DOI: $10.34103 / A R G U M E N T U M / 2021 / 43$

\subsubsection{Le cas spécial de la scène intercalée}

Dans la nouvelle analysée, nous avons remarqué une spécificité de la structure scénique : c'est notamment le cas de la scène intercalée. Au sein de la Scène 1 - celle-ci comprenant la longue description de la procession, des jeunes mariés, des invités, du paysage et de la grande ferme nous avons pu détecter une scène intercalée dont le thème est les coups de fusil.

Lorsqu'ils tournèrent la grande barrière de la ferme maritale, quarante coups de fusil éclatèrent sans qu'on vît les tireurs cachés dans les fossés. A ce bruit, une grosse gaieté saisit les hommes qui gigotaient lourdement en leurs habits de fête ; et Patu, quittant sa femme, sauta sur un valet qu'il apercevait derrière un arbre, empoigna son arme, et lâcha lui-même un coup en gambadant comme un poulain. (63)

Le point de rupture marquant le début de cette scène intercalée est indiqué par la conjonction de temps lorsque, tandis que le retour à la scène englobante - c'est-à-dire à la Scène 1 - est signalé par l'adverbe de temps puis.

Puis on se remit en route $[\ldots](63)$

La scène intercalée interrompt le récit de la procession, et nous invite à examiner le rôle spécifique qu'elle accomplit. Il est à voir comment elle se rattache à la structure scénique, et au déroulement du récit.

Le motif des coups de fusil est, selon la terminologie de Gérard Genette, une prolepse. ${ }^{11} \mathrm{Ce}$ motif prépare un événement postérieur, à savoir l'apparition des «braconniers » imaginaires qui sont, en réalité, les quatre gars faisant farce au jeune mari. Ce moment postérieur sera indiqué de la même manière, par un coup de feu, à la frontière entre la Scène 4 et la Scène 5 : "[...] quand un coup de feu retentit au loin » (66), et sera renforcé par un deuxième coup de feu : "[...] une nouvelle détonation, plus proche celle-là, retentit. » (67)

Le motif des coups de fusil, et celui de la gaieté des invités peuvent être considérés comme des amorces, ${ }^{12}$ selon la terminologie de G. Genette.

Le motif des coups de fusil est mentionné deux fois dans la Scène 1 : il apparaît d'abord dans la scène intercalée, puis il sera repris encore une fois : " [...] et les coups de fusil ne cessaient pas, éclatant de tous les côtés à la fois, mêlant à l'air une buée de poudre [...] » (64). Ces apparitions des coups de fusil dans la Scène 1 préparent, sans les annoncer, les coups de fusil de la Scène 5, ces derniers faisant soupçonner cette fois l'arrivée des «braconniers ».

L'évocation de la gaieté, à plusieurs reprises le long du texte, préfigure la farce commise contre le jeune mari. Ses représentations lexicales se montrent variées : une grosse gaieté; les lourdes plaisanteries; des grivoiseries; un rire ; des hurlements de gaieté ; des farces ; la joie ; une pluie de polissonneries à double sens.

La mention des « braconniers » par l'un des gars dans la Scène 3 représente un autre cas de prolepse : c'est la préfiguration de la farce, de l'épisode qui aura lieu - au niveau de l'histoire - entre la Scène 5 et la Scène $6:$ l'action réelle des « braconniers » pendant la nuit de noces.

11 Gérard Genette utilise le terme prolepse - un terme plus neutre, dans sa conception, pour l'anticipation : il désigne par prolepse «toute manœuvre narrative consistant à raconter ou évoquer d'avance un événement ultérieur [...] au point de l'histoire où on se trouve » (Genette 1972 : 82).

12 Les amorces, dans la terminologie genettienne, sont de « simples pierres d'attente sans anticipation, même allusive, qui ne trouveront leur signification que plus tard et qui relèvent de l'art tout classique de la „préparation” » (Genette 1972 : 112). 
Beatrix Babett Bódi:

La problématique de la scène et du segment dans « Farce normande » de Guy de Maupassant

Argumentum 17 (2021), 807-819

Debreceni Egyetemi Kiadó

DOI: $10.34103 / A R G U M E N T U M / 2021 / 43$

« C'est les braconniers qui vont s'en donner c'te nuit, avec la lune qu'y a !... Dis donc, Jean, c'est pas c'te

lune-là qu' tu guetteras, toi ? »

Le marié, brusquement, se tourna :

«Qu'i z’y viennent, les braconniers!»

Mais l'autre se mit à rire :

«Ah! i peuvent y revenir ; tu quitteras pas ta besogne pour ça !» (65)

Ces amorces peuvent être détectées par le lecteur attentif, mais leur fonction ne prend sens qu'au moment où la farce est accomplie - c'est-à-dire vers la fin de la nouvelle. ${ }^{13}$

\subsection{Une analyse actantielle - les segments}

Pour examiner la distribution des rôles actantiels - dans les cadres d'une deuxième lecture -, nous allons procéder selon le modèle de A. J. Greimas. Comme nous l'avons déjà mentionné, l'examen de la distribution des rôles actantiels nous permettra de dresser la structure actantielle de la nouvelle, et en même temps, de définir la segmentation.

\subsubsection{La structure actantielle}

La structure de la nouvelle présente deux segments. Dans le premier segment, qui offre la situation initiale, nous pouvons constater une réciprocité dans la distribution de certains rôles actantiels. En effet, les protagonistes de l'histoire, le jeune marié, Jean Patu et sa mariée, Rosalie, sont représentés mutuellement dans les rôles de sujet, objet et destinataire. ${ }^{14}$ La raison en est que la force d'action, qui a un effet sur la dynamique de l'intrigue, est leur désir mutuel de se marier, désir qui prend donc le rôle de l'actant destinateur. Les invités réalisent l'actant adjuvant, car tout le monde est heureux pour les jeunes mariés. L'actant opposant est représenté par les quatre gars qui font des plaisanteries ; leur manifestation lexicale par des noms communs suggère que n'importe quel homme pourrait prendre ce rôle parmi les invités. Dans ce premier segment, les quatre gars sont présents « sur scène », mais ils ne sont pas encore très actifs, en tant qu'opposants, sauf qu'ils prononcent des paroles qui font rire les hommes et rougir la mariée. Pourtant, leurs propos évoquant les « braconniers » peuvent les rendre suspects, de sorte que l'on pourrait qualifier ici ces acteurs d'« opposants virtuels », non encore révélés.

Le tournant qui annonce le deuxième segment, plus dramatique, est marqué - au niveau des scènes - par les deux coups de feu consécutifs interrompant la nuit de noces : ces coups de feu signalent l'action des « braconniers » imaginaires - en apparence un nouveau groupe d'acteurs mais en réalité les quatre gars -, causant par là un changement dans la distribution des rôles actantiels.

Ainsi, dans le deuxième segment, le sujet, c'est le jeune marié, Jean Patu. L'objet est représenté par l'acteur non animé et abstrait : l'intention de Jean de protéger ses biens (menacés par des « braconniers » imaginaires). L'actant destinateur est figuré par le même acteur non animé et abstrait : l'intention de Jean de protéger ses biens. Le destinataire est Jean lui-même.

13 «A la différence de l'annonce, l'amorce n'est donc en principe, à sa place dans le texte, qu'un ,germe insignifiant", et même imperceptible, dont la valeur de germe ne sera reconnue que plus tard, et de façon rétrospective. Encore faut-il tenir compte de l'éventuelle (ou plutôt variable) compétence narrative du lecteur, née de l'habitude, qui permet de déchiffrer de plus en plus vite le code narratif général, ou propre à tel genre, ou à telle œuvre, et d'identifier les ,germes” dès leur apparition » (Genette 1972 : 113).

14 Pour ce syncrétisme, v. plus haut, note 10. 
L'actant adjuvant reste vide dans ce segment, car nous ne pouvons pas détecter d'acteur incarnant cet actant. En revanche, plusieurs acteurs prennent le rôle de l'opposant : Rosalie, le désir - acteur non animé - et les « braconniers » imaginaires.

La mise en scène des personnages-acteurs a un effet sur le découpage du texte et de l'histoire à la fois : le changement de l'acteur représentant l'actant destinateur implique la redistribution des rôles. Les forces d'action changent, et cela entraîne le changement de la structure actantielle, et en même temps, marque le début du deuxième segment.

Dans la nouvelle analysée, avec une redistribution des rôles, nous avons repéré deux segments constituant la structure actantielle; en résumé, nous pouvons donc établir que cette nouvelle est un exemple des nouvelles à structure actantielle variable.

\begin{tabular}{|c|c|c|c|c|}
\hline & Actant & Acteur & Actant & Acteur \\
\hline \multirow[t]{4}{*}{ Premier segment } & Sujet & Jean Patu, Rosalie & Objet & Rosalie, Jean Patu \\
\hline & Destinateur & $\begin{array}{l}\text { le désir de se } \\
\text { marier et de } \\
\text { s'amuser aux fêtes } \\
\text { des noces }\end{array}$ & Destinataire & Jean Patu, Rosalie \\
\hline & Adjuvant & les invités & $\begin{array}{l}\text { Opposant } \\
\text { (virtuel) }\end{array}$ & les quatre gars \\
\hline & Actant & Acteur & Actant & Acteur \\
\hline \multirow[t]{3}{*}{ Deuxième segment } & Sujet & Jean Patu & Objet & $\begin{array}{l}\text { protéger ses biens menacés par } \\
\text { les « braconniers » imaginaires } \\
\text { (les quatre gars) }\end{array}$ \\
\hline & Destinateur & $\begin{array}{l}\text { le besoin de } \\
\text { protéger ses biens }\end{array}$ & Destinataire & Jean Patu \\
\hline & Adjuvant & - & Opposant & $\begin{array}{l}\text { Rosalie ; le désir ; les } \\
\text { «braconniers » (les quatre gars) }\end{array}$ \\
\hline
\end{tabular}

Tableau 2 représentant la distribution des rôles actantiels

\subsubsection{Cas spécial : Les éléments abstraits de la structure actantielle}

Nous avons identifié deux acteurs non animés et abstraits : le désir dans le premier segment, et l'intention du héros de protéger ses biens dans le deuxième segment. Les deux acteurs représentent l'actant destinateur; ce sont des forces d'action qui régissent les relations des personnages, et par conséquent, ont un impact incontestable sur le dynamisme de l'intrigue.

Ce dynamisme est caractérisé aussi par une transformation état d'équilibre - état de déséquilibre - nouvel état d'équilibre, qui est en corrélation avec les changements de la structure actantielle. 


\subsubsection{Cas spécial : être et paraître}

Le thème de l'être et du paraître apparaît dans la nouvelle sous forme des «braconniers » imaginaires : ils représentent l'actant opposant du deuxième segment. Ils font référence à un péril imaginaire, et sont incarnés par les quatre gars, amis du jeune mari, lui faisant farce.

Bien que le héros se trouve dans une situation absurde - en effet, il ne sait pas comment protéger ses biens d'un péril imaginaire (mais qu'il croit réel) - un état d'équilibre est rétabli à la fin, car une fois la farce accomplie, le jeune mari est finalement retourné à sa femme en bonne santé et en bonne humeur.

\subsection{Relation des deux structures}

Nous venons de décrire deux systèmes correspondant à des niveaux différents de la nouvelle. Reste à voir comment les deux types d'unités fonctionnent dans les cadres d'une même œuvre littéraire. Est-ce que ce sont des unités parallèles, ou peut-on détecter une corrélation entre elles ? S'agit-il d'unités dont les limites coïncident ou non ?

Dans la nouvelle analysée, nous avons trouvé que les scènes ne coïncident pas nécessairement avec les segments déterminés par la structure actantielle. Un segment peut être une unité éventuellement plus vaste, et peut alors englober plusieurs scènes. C'est le cas du premier segment englobant les quatre premières scènes, et du deuxième segment englobant les deux dernières scènes.

Dans le cas d'une œuvre narrative littéraire, où la distribution des rôles actantiels reste le même tout au long de l'histoire, les mêmes personnages (ou acteurs) accomplissent les mêmes rôles actantiels. Il s'agit là d'une structure simple, avec une distribution fixe des rôles qui régit l'histoire, et constitue, dans ce cas, un seul segment. Or, au sein de ce même segment, plusieurs scènes peuvent se succéder.

Au cas où la distribution des rôles actantiels change au cours de l'histoire, nous observons plusieurs segments et plusieurs scènes au sein du même texte narratif. Mais il est à noter qu'une nouvelle scène ne marque pas nécessairement le début d'un nouveau segment, tandis que le début d'un nouveau segment coïncide normalement avec le début d'une nouvelle scène.

Les deux passages du texte qui se trouvent à la fin de la nouvelle, la cloture et le bref commentaire de la part du narrateur, ne font partie intégrante ni de la structure scénique, ni de la structure actantielle. La clôture donne un certain dénouement, en vue d'atténuer le caractère sérieux de la situation, et montrant que le mari a compris la farce : "Oh! pour une farce! c'était une bonne farce. " (67) Le bref commentaire final de la part du narrateur, à caractère généralisant, est là à titre de « moralité ». 
Beatrix Babett Bódi:

La problématique de la scène et du segment dans « Farce normande » de Guy de Maupassant Argumentum 17 (2021), 807-819

Debreceni Egyetemi Kiadó

DOI: 10.34103/ARGUMENTUM/2021/43

\begin{tabular}{|l|l|l|}
\hline Scènes & $\begin{array}{l}\text { Thème de la scène } \\
\text { Scène } 1\end{array}$ & Segments \\
\hline Scène 2 & Le dîner & Premier segment \\
\hline Scène 3 & Les plaisanteries verbales à la table de dîner & \\
\hline Scène 4 & La nuit de noces dans la chambre des jeunes mariés & \\
\hline Scène 5 & L'apparition des « braconniers » & Deuxième segment \\
\hline Scène 6 & Le mari retrouvé & \\
\hline
\end{tabular}

Tableau 3 représentant la relation des deux structures

\section{Conclusion}

Dans notre étude, nous avons observé dans « Farce normande » de Maupassant l'organisation du texte narratif littéraire en fonction de la structure scénique et de la structure actantielle. Nous avons cherché à définir les notions de scène et de segment, sous-unités du texte narratif littéraire, et, dans les cadres d'une analyse textuelle et narratologique, nous avons identifié les éléments de la structure scénique, et ceux de la structure des segments.

Nous avons trouvé que la structure scénique définit la composition de la nouvelle au niveau de surface, alors que la structure actantielle détermine le niveau profond du texte.

En guise de conclusion, nous pouvons constater que, dans cette nouvelle, les personnages ou groupes de personnages - caractérisent les scènes individuelles, et, dans certains cas, peuvent définir le changement de scène. La délimitation des scènes consécutives est marquée surtout par des indices textuels concrets : par le changement d'espace, par l'emploi d'adverbes et d'autres compléments circonstanciels de lieu et de temps, ou bien par le changement des temps verbaux. Ces marqueurs textuels peuvent s'appliquer individuellement ou en combinaisons variées.

La mise en scène des personnages-acteurs a un effet sur l'organisation actantielle : le changement de l'acteur représentant le destinateur implique la modification des forces d'action et la redistribution des rôles actantiels, et par cela, entraîne aussi la modification de la segmentation.

Compléter cette analyse par l'analyse d'autres nouvelles sur un corpus assez large, nous permettra d'identifier d'éventuelles règles de segmentation et d'établir une typologie des nouvelles analysées. 


\title{
Bibliographie
}

Texte analysé

Maupassant, Guy de (1959) : Farce normande. In : Maupassant, Guy de : Contes et nouvelles I. Texte établi par A.-M. Schmidt. Paris : Éditions Albin Michel, 63-67.

\section{Études}

Adam, J.-M. (1992) : Les textes : types et prototypes. Paris : Éditions Nathan.

Combettes, B. (1983) : Pour une grammaire textuelle. La progression thématique. Bruxelles : Éditions A. De Boeck \& Paris-Gembloux : Éditions J. Duculot.

Genette, G. (1972) : Discours du récit. In : Genette : Figures III. Paris : Éditions du Seuil, 65282.

Greimas, A. J. (1966) : Réflexions sur les modèles actantiels. In: Greimas : Sémantique structurale. Paris : Larousse, 172-186.

Greimas, A. J. (1970) : La structure des actants du récit. In : Greimas : Du sens. Essais sémiotiques. Paris : Éditions du Seuil, 249-270.

Greimas, A. J. (1976) : Maupassant. La sémiotique du texte : exercices pratiques. Paris : Éditions du Seuil.

Maingueneau, D. (2003) : Linguistique pour le texte littéraire. Paris : Nathan.

Riegel, M., Pellat, J.-Ch. \& Rioul, R. (2009) : Grammaire méthodique du français. Paris : Presses Universitaires de France ( $7^{\mathrm{e}}$ édition revue et augmentée, $1^{\text {ère }}$ édition : 1994).

Ubersfeld, A. (1982) : Lire le théâtre. Paris : Messidor / Éditions Sociales.

\author{
Beatrix Babett Bódi \\ Université de Debrecen \\ École Doctorale de Linguistique \\ Département de Langue Française \\ H-4002 Debrecen \\ Pf. 400 \\ bodibabett@hotmail.com
}

